DOI: 10.1002/zaac.200670082

\title{
Nichtharmonische Strukturverfeinerung an ionenleitenden Silbermineralien
}

\author{
Michael Leitl, Arno Pfitzner* \\ Institut für Anorganische Chemie, Universität Regensburg, \\ Universitätsstraße 31, D-93040 Regensburg
}

Keywords: Pyrargyrite; Stephanite; Pearceite; Ionic conductivity; Impedance spectroscopy; Non-harmonic refinement

Silbermineralien sind für ihre interessanten strukturellen und physikalischen Eigenschaften bekannt. $\mathrm{Ag}_{3} \mathrm{SbS}_{3}$ und $\mathrm{Ag}_{3} \mathrm{AsS}_{3}[1,2]$ zum Beispiel sind beide Halbleiter und gute Ionenleiter. Einige dieser Mineralien errangen technisches Interesse durch ihre interessanten Eigenschaften im Bereich der optischen Anwendungen. So ist $\mathrm{Ag}_{3} \mathrm{AsS}_{3}$ bekannt dafür, dass es gute nichtlinear-optische Eigenschaften besitzt.

Viele Silbermineralien wurden bisher nur unzureichend strukturell charakterisiert, da die Beschreibung der Silberverteilung mittels anisotroper Auslenkungsparameter in ionenleitenden Verbindungen oft kein befriedigendes Ergebnis liefert. Ein Beispiel hierfür geben Evain et al. [3]. Um die Struktur-Eigenschafts-Beziehungen für diese ionenleitenden Verbindungen besser verstehen zu können, wurden die Kristallstrukturen von synthetischem Pyrargyrit $\mathrm{Ag}_{3} \mathrm{SbS}_{3}$ (Abb. 1), natürlichem Stephanit $\mathrm{Ag}_{5} \mathrm{SbS}_{4}$ (Leihgabe der Mineralogischen Staatssammlung München, Sammlungsnummer 51164) und synthetischem Pearceit $\left(\mathrm{Ag}_{1-x} \mathrm{Cu}_{x}\right)_{16} \mathrm{As}_{2} \mathrm{~S}_{11}(x=0.26$, 0.32) unter Verwendung nichtharmonischer Auslenkungsparameter verfeinert und mit den Ergebnissen impedanzspektroskopischer Untersuchungen (Abb. 2) in Beziehung gesetzt.

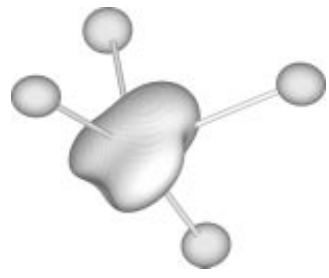

Abb. $1 \mathrm{AgS}_{4}$-Einheit in der

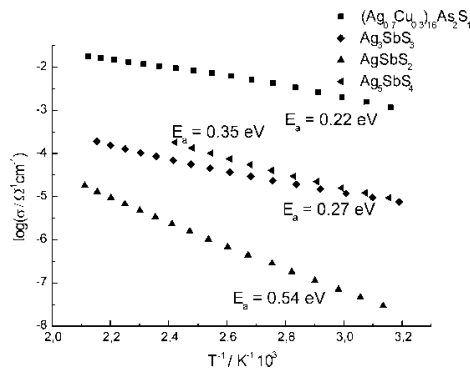

Abb. 2 Leitfähigkeiten von Myargyrit $\mathrm{AgSbS}_{2}$, Pyrargyrit $\mathrm{Ag}_{3} \mathrm{SbS}_{3}$, Stephanit $\mathrm{Ag}_{5} \mathrm{SbS}_{4}$ und Pearceit $\left(\mathrm{Ag}_{1-x} \mathrm{Cu}_{x}\right)_{16} \mathrm{As}_{2} \mathrm{~S}_{11}$

[1] P. Engel, W. Nowacki, Neues Jahrb. Mineral. Mh. 1966, 181.

[2] K. A. Schönau, S. A. T. Redfern, J. Appl. Phys. 2002, 92, 7415.

[3] M. Evain, E. Gaudin, F. Boucher, V. Petrícek, F. Taulelle, Acta Cryst. B, 1998, 54, 376. 